\title{
AMBIGUITIES IN THE INTERPRETATION OF TIME-RESOLVED FLUORESCENCE ANISOTROPY MEASUREMENTS ON LIPID VESICLE SYSTEMS
}

\author{
H. VAN LANGEN, Y.K. LEVINE \\ Department of Molecular Biophysics, Physics Laboratory, Princetonplein 5, 3584 CC Utrecht, The Netherlands \\ M. AMELOOT \\ Limburgs Universttair Centrum, Division of Biophysics, Universitaire Campus, 3610 Diepenbeek, Belgium
}

and

\author{
H. POTTEL \\ Interdisciplinair Research Centrum, Katholieke Universiteit Leuven, Campus Kortrijk, 8500 Kortrijk, Belgium
}

Received 13 July 1987

\begin{abstract}
Analysis of time-resolved fluorescence anisotropy measurements on DPH and TMA-DPH in POPC vesicles with and without cholesterol in terms of the rotational diffusion model shows two distinct $\chi_{\mathrm{r}}^{2}$ minima which are statistically equivalent. This is explained by the fact that the anisotropy decay function is given by a sum of three correlation functions which cannot be uniquely separated into individual contributions. The two solutions yield contradictory results for the effect of cholesterol on the probe dynamics. It is shown that the Maier-Saupe potential and the "wobble-in-cone" model do not give an adequate picture of the orientational order and the reorientational dynamics.
\end{abstract}

\section{Introduction}

Lipid vesicles are widely used as model systems in the study of the dynamic structure of lipid bilayer membranes. Much of our understanding of these systems has been obtained from probe techniques which monitor the molecular order and dynamics of extraneous molecules embedding in the membrane structure $[1,2]$. In particular, time-dependent fluorescence anisotropy measurements provide a convenient means of studying membrane dynamics as the reorientational motions of the probe molecules take place on the timescale of the intrinsic fluorescence decay $[1,3,4]$. The fluorescent molecules 1,6diphenyl-1,3,5-hexatriene (DPH) and its polar analogue trimethylamino-DPH (TMA-DPH) are often used in experiments.

The main difficulty with such experiments is that the anisotropy decay function characterizing the reorientational motions cannot be obtained directly from the experimentally determined time-dependent signals $[1,3,5]$. The anisotropy has to be extracted numerically from the observed decays, usually with non-linear least-squares reiterative deconvolution techniques [5,6]. This, however, can only be achieved by using a mathematical expression for the anisotropy decay which can be done in two ways. In a model-independent treatment, the time-dependent anisotropy is described as a multi-exponential decay with a rest anisotropy term [4]. The problem with this type of analysis is relating the parameters obtained with physical processes. For this reason many workers prefer to analyze the experimental data in terms of physical models for the rotational motion of the probe molecules $[1,3,7,8]$.

Here we report an analysis of the anisotropy decay of DPH and TMA-DPH molecules in vesicles of palmitoyl-oleoyl phosphatidylcholine (POPC) in terms of the rotational diffusion model that yields two distinct solutions which are statistically equivalent. It is 
shown that this ambiguity arises directly from the fact that the anisotropy decay is given by a sum over a number of orientational time-correlation functions $[1,7,8]$, which cannot be uniquely resolved into individual contributions.

\section{Theoretical considerations}

The time-dependent polarized fluorescence intensities observed at time $t$ after excitation with a $\delta$ function pulse of plane-polarized light at time $t=0$ are given by $[1,4,6-8]$

$$
\begin{aligned}
& I_{\|}(t)=\frac{1}{3} F(t)[1+2 r(t)], \\
& I_{\perp}(t)=\frac{1}{3} F(t)[1-r(t)],
\end{aligned}
$$

where $F(t)$ is the intrinsic fluorescence decay function and $r(t)$ is the anisotropy decay function. Here $I_{\|}$and $I_{\perp}$ respectively denote the intensities of light polarized parallel and perpendicular to the polarization direction of the exciting light.

In the case of a molecule, such as DPH and TMA-DPH, which can be considered to possess an effective cylindrical symmetry and an absorption transition moment lying along the molecular symmetry axis [1,3-9], the anisotropy decay can be written as a sum of three correlation functions $G_{k}(t)$ $[1,7,8]$.

$$
r(t)=r(0)\left[G_{0}(t)+2 G_{1}(t)+2 G_{2}(t)\right]
$$

with

$$
G_{k}(t)=\left\langle D_{k 0}^{2}\left(\Omega_{0}\right) D_{k 0}^{2 *}\left(\Omega_{t}\right)\right\rangle, \quad k=0,1,2 .
$$

Here $D_{m n}^{2}$ are Wigner rotation matrix elements $[1,10]$ and $\Omega_{0}$ and $\Omega_{t}$ denote respectively the orientation of the long molecular axis at time $t=0$ and time $t$. The initial values of the correlation functions $G_{k}(0)$ are given by linear combinations of the order parameters $\left\langle P_{2}\right\rangle=\frac{1}{2}\left\langle 3 \cos ^{2} \beta-1\right\rangle$ and $\left\langle P_{4}\right\rangle=$ $\frac{1}{8}\left\langle 35 \cos ^{4} \beta-30 \cos ^{2} \beta+3\right\rangle[1,7,8]$.

The time behaviour of $G_{k}(t)$ can be obtained numerically within the framework of the rotational diffusion model $[1,11]$ on specifying the orienting potential $U(\beta)$ :

$$
U(\beta)=k T\left[\lambda_{2} P_{2}(\cos \beta)+\lambda_{4} P_{4}(\cos \beta)\right],
$$

where $\beta$ is the angle between the long molecular axis and the normal to the local bilayer surface. $P_{L}(\cos \beta)$ is the Legendre polynomial of order $L$. The pair of coefficients $\lambda_{2}$ and $\lambda_{4}$ uniquely defines a pair of $\left\langle P_{2}\right\rangle$ and $\left\langle P_{4}\right\rangle$ values [12-14]. Restriction of the potential given by eq. ( 4$)$ to the first term only yields the so-called Maier-Saupe potential. In general the correlation functions $G_{k}(t)$ are given by a sum of exponential decays $[1,11]$

$G_{k}(t)=\sum_{m=0}^{\infty} b_{m}^{k} \exp \left(-\alpha_{m}^{k} D_{\perp} t\right)$,

where the amplitudes and decay rates $b_{m}^{k}$ and $\alpha_{m}^{k}$ are determined by $\lambda_{2}$ and $\lambda_{4}$. In many practical situations, however, it is found that $G_{k}(t)$ is essentially monoexponential $[1,11]$,

$G_{k}(t)=\left[G_{k}(0)-G_{k}(\infty)\right] \exp \left(-\alpha_{1}^{k} D_{\perp} t\right)+G_{k}(\infty)$,

$G_{h}(\infty)=\left\langle P_{2}\right\rangle^{2} \delta_{k 0}$,

where $\alpha_{1}^{k}$ is the smallest decay term of the numerical solution to eq. (5).

\section{Experimental}

\subsection{Sample preparation}

The probe molecules DPH and TMA-DPH were purchased respectively from Fluka AG and Molecular Probes Inc. and used as received. Fresh stock solutions of the probes in ethanol $\left(5 \times 10^{-4} \mathrm{M}\right)$ were kept in darkness at $4^{\circ} \mathrm{C}$. Cholesterol and POPC were purchased from Sigma Chemical Co. and used without further purification. The lipids were dissolved in a mixture of ethanol and freshly doubly distilled chloroform. Solutions of the probe molecules were added as necessary to give a lipid:probe molecular ratio of 250:1. Non-labelled samples were also prepared for control experiments. After evaporating the solvent the lipids were dispersed in $6 \mathrm{ml}$ of a buffer containing $2 \times 10^{-2} \mathrm{M}$ Tris and $7.5 \times 10^{-6} \mathrm{M}$ EDTA at $p H$ 8.0. Vesicles were formed by sonicating the aqueous dispersion in the dark under a nitrogen atmosphere. Any large liposome remnants were removed by centrifuging for $1 \mathrm{~h}$ at $150000 \mathrm{~g}$. The final vesicle suspensions contained $1.3 \pm 0.2 \mathrm{mg} \mathrm{lipid} / \mathrm{ml}$ buffer solution determined as described by Chen et 
al. [15]. These samples were kept in darkness at $4^{\circ} \mathrm{C}$ for not more than a week prior to the experiments.

\subsection{Time-resolved measurements}

The time-resolved anisotropy decay measurements were carried out with the Synchrotron Radiation Source (SRS) in Daresbury, UK, operating in single-bunch mode. A standard single-photon counting system [5] was used. The start pulse was obtained from a ring pulse generated by the circulating $2 \mathrm{GeV}$ electrons. The first photon detected by the PMT (Philips, XP2020Q) provided the stop pulse for the time-to-pulse-height converter (TPHC, Ortec 457). The output pulses from the TPHC were digitized and stored in a multichannel analyzer (MCA, I/T Techno Inc. 5400) using 1024 channels for each recorded decay. The standard $90^{\circ}$-scattering geometry was used. The probes were excited at $358 \pm 5 \mathrm{~nm}$ (Spex monochromator) and the fluorescence emission was passed through a GG 395 low cut-off filter (Schott) and a Balzers interference filter, $438 \pm 7 \mathrm{~nm}$, before incidence on the PMT. The excitation functions, needed for the numerical deconvolution procedures, were obtained from elastic scattering by vesicle suspensions at the emission wavelength. In all the experiments the count rates were kept below $1 \%$ of the excitation pulse repetition rate in order to avoid problems due to pile-up. The intrinsic fluorescence decay of the probes was measured under the same experimental conditions, setting the analyzer axis at $54.7^{\circ}$ with respect to the vertical, the direction of polarization of the exciting beam. The temperature was maintained at $20 \pm 2^{\circ} \mathrm{C}$ throughout.

\subsection{Numerical analysis}

Non-linear least-squares reiterative deconvolution methods [5] were used in the analysis of the experimental decay curves. The least-squares optimization was carried out with the ZXSSQ routine from the IMSL library. This routine utilizes a Levenberg -Marquardt minimization algorithm. The decay curves $I_{\|}(t)$ and $I_{\perp}(t)$ were analyzed simultaneously [5].

\section{Results and discussion}

Analysis of the experiments performed at the magic angle indicated that the intrinsic fluorescence decay of both DPH and TMA-DPH in POPC vesicles with and without cholesterol can be described by a biexponential

$F(t)=\alpha_{1} \exp \left(-t / \tau_{1}\right)+\alpha_{2} \exp \left(-t / \tau_{2}\right)$

which requires the optimization of four parameters. Four parameters are also necessary to describe the time-dependent anisotropy $r(t)$, eqs. (2)-(6). Here $r(0), D_{\perp}, \lambda_{2}$ and $\lambda_{4}$ enter as adjustable parameters. Fig. 1 shows an example of the simultaneous analysis of the experimentally determined intensity decays. Surprisingly, the analysis of the results for every system studied revealed the existence of two distinct reduced $\chi^{2}$ minima. The minimum located in the numerical deconvolution procedure was found to be strongly dependent on the start values assigned to the four parameters describing $r(t)$. The model parameters obtained in the analyses are summarized in table 1. Interestingly the errors for minimum II are larger than those for $I$. The double minimum is also found when the initial anisotropy $r(0)$ is in the range $0.40-0.38$. In these cases significant higher $\chi_{\mathrm{r}}^{2}$ values and worse autocorrelations of the residuals are found at the minima. It can be seen from table 1 that both minima yield virtually the same intrinsic fluorescence decay, so that the effect can be ascribed wholly to the behaviour of the anisotropy decay. Indeed we see that the two solutions correspond to a completely different ordering of the probe molecules. This is illustrated in table 2 in terms of the order parameters $\left\langle P_{2}\right\rangle$ and $\left\langle P_{4}\right\rangle$. Solution II appears to yield similar order parameters to those expected from the "wobble-in-cone" model [3,7]. The small differences in the values of $\left\langle P_{2}\right\rangle$ obtained arise simply because the weighted least-squares method conserves the area under the measured curve [16], so that eq. (1) requires that $\int_{0}^{\infty} F(t) r(t) \mathrm{d} t$ be an experimental constant.

In order to understand the role of the anisotropy decay function $r(t)$ in the calculations, we have repeated the analysis in a systematic way by keeping the order parameters $\left\langle P_{2}\right\rangle$ and $\left\langle P_{4}\right\rangle$ constant during the deconvolution procedure. In these calculations $\left\langle P_{2}\right\rangle$ was fixed at a value in the range found, 


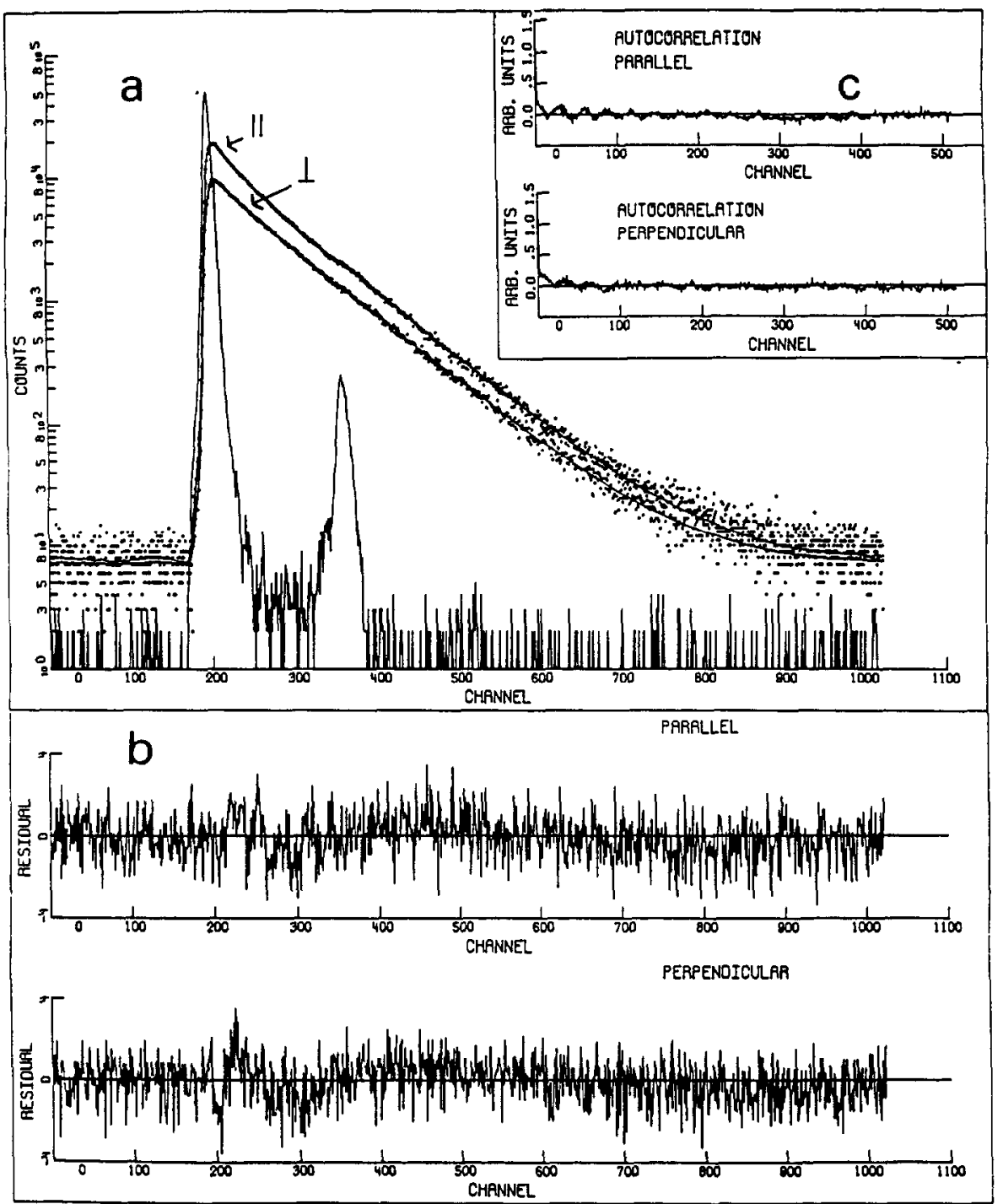

Fig. 1. Analysis of the time-resolved anisotropy for vesicles of POPC with cholesterol and TMA-DPH. The dots in (a) represent the experimental data while the continuous lines give the best simultaneous fit. The narrow excitation pulse of the synchrotron and the after pulse caused by the PMT are also shown. (b) The residuals for both components of the fit and (c) the autocorrelation functions of these residuals. 1 channel equals 80 ps.

table 2 , and the value of $\left\langle P_{4}\right\rangle$ was varied within its physically allowed range [12-14].

An example of the dependence of the minimal $\chi_{r}^{2}$ value of $\left\langle P_{4}\right\rangle$ is shown in fig. 2 . All the systems studied exhibited this double-well behaviour. Interestingly, the solution yielding the highest $\chi_{\mathrm{r}}^{2}$ value between the two wells is given by a $\left\langle P_{4}\right\rangle$ value which closely corresponds to the prediction of the Maier-Saupe potential. This finding indicates the inadequacies of this potential for the description of the reorientational motions of these probe molecules, in agreement with our earlier conclusions from steady-state studies of oriented multilayer systems $[12,13]$ and from studies of vesicles of saturated lipids [6]. The origin of this behaviour appears to lie in the fact that the anisotropy decay $r(t)$, eq. (2), is given by a sum over three correlation functions. Our calculations show that the same sum can be resolved 
Table 1

Recovered fit parameters for the $\chi_{\mathrm{r}}^{2}$ minima

\begin{tabular}{|c|c|c|c|c|c|c|c|c|c|c|}
\hline & $\chi_{r}^{2}$ & $\alpha_{1}$ & $\begin{array}{l}\tau_{1} \\
\text { (ns) }\end{array}$ & $\alpha_{2}$ & $\begin{array}{l}\tau_{2} \\
(\mathrm{~ns})\end{array}$ & $r(0)$ & $\lambda_{2}$ & $\lambda_{4}$ & $\begin{array}{l}D_{\perp} \\
\left(\mathrm{ns}^{-1}\right)\end{array}$ & $\begin{array}{l}\left\langle\tau_{F}\right\rangle^{\text {a) }} \\
\text { (ns) }\end{array}$ \\
\hline \multicolumn{11}{|l|}{$\overline{\text { POPC }}$} \\
\hline $\begin{array}{l}\text { I DPH } \\
\text { errors }\end{array}$ & 1.134 & $\begin{array}{l}0.17 \\
0.005\end{array}$ & $\begin{array}{l}4.13 \\
0.02\end{array}$ & $\begin{array}{l}0.83 \\
0.005\end{array}$ & $\begin{array}{l}9.15 \\
0.03\end{array}$ & $\begin{array}{l}0.30 \\
0.01\end{array}$ & $\begin{array}{r}0.1 \\
0.1\end{array}$ & $\begin{array}{r}-2.2 \\
0.1\end{array}$ & $\begin{array}{l}0.107 \\
0.007\end{array}$ & $\begin{array}{l}8.73 \\
0.02\end{array}$ \\
\hline $\begin{array}{l}\text { Il DPH } \\
\text { errors }\end{array}$ & 1.142 & $\begin{array}{l}0.17 \\
0.005\end{array}$ & $\begin{array}{l}4.20 \\
0.02\end{array}$ & $\begin{array}{l}0.83 \\
0.005\end{array}$ & $\begin{array}{l}9.14 \\
0.03\end{array}$ & $\begin{array}{l}0.30 \\
0.01\end{array}$ & $\begin{array}{r}-1.6 \\
0.2\end{array}$ & $\begin{array}{l}4.1 \\
1.0\end{array}$ & $\begin{array}{l}0.125 \\
0.023\end{array}$ & $\begin{array}{l}8.72 \\
0.02\end{array}$ \\
\hline $\begin{array}{l}\text { I TMA } \\
\text { errors }\end{array}$ & 1.464 & $\begin{array}{l}0.36 \\
0.005\end{array}$ & $\begin{array}{l}1.40 \\
0.02\end{array}$ & $\begin{array}{l}0.64 \\
0.005\end{array}$ & $\begin{array}{l}4.45 \\
0.03\end{array}$ & $\begin{array}{l}0.32 \\
0.01\end{array}$ & $\begin{array}{r}-1.2 \\
0.1\end{array}$ & $\begin{array}{r}-2.1 \\
0.2\end{array}$ & $\begin{array}{l}0.089 \\
0.007\end{array}$ & $\begin{array}{l}4.00 \\
0.02\end{array}$ \\
\hline $\begin{array}{l}\text { II TMA } \\
\text { errors }\end{array}$ & 1.469 & $\begin{array}{l}0.35 \\
0.005\end{array}$ & $\begin{array}{l}1.36 \\
0.02\end{array}$ & $\begin{array}{l}0.65 \\
0.005\end{array}$ & $\begin{array}{l}4.43 \\
0.03\end{array}$ & $\begin{array}{l}0.31 \\
0.01\end{array}$ & $\begin{array}{r}-7.7 \\
2.0\end{array}$ & $\begin{array}{l}4.2 \\
1.5\end{array}$ & $\begin{array}{l}0.072 \\
0.024\end{array}$ & $\begin{array}{l}3.99 \\
0.02\end{array}$ \\
\hline \multicolumn{11}{|c|}{ cholesterol added ( $25 \%$ by weight) } \\
\hline $\begin{array}{l}\text { I DPH } \\
\text { errors }\end{array}$ & 1.294 & $\begin{array}{l}0.10 \\
0.005\end{array}$ & $\begin{array}{l}4.99 \\
0.02\end{array}$ & $\begin{array}{l}0.90 \\
0.005\end{array}$ & $\begin{array}{l}9.99 \\
0.04\end{array}$ & $\begin{array}{l}0.29 \\
0.01\end{array}$ & $\begin{array}{r}-1.1 \\
0.1\end{array}$ & $\begin{array}{r}-2.2 \\
0.2\end{array}$ & $\begin{array}{l}0.116 \\
0.008\end{array}$ & $\begin{array}{l}9.73 \\
0.03\end{array}$ \\
\hline $\begin{array}{l}\text { II DPH } \\
\text { errors }\end{array}$ & 1.343 & $\begin{array}{l}0.11 \\
0.005\end{array}$ & $\begin{array}{l}5.44 \\
0.02\end{array}$ & $\begin{array}{l}0.89 \\
0.005\end{array}$ & $\begin{array}{r}10.01 \\
0.04\end{array}$ & $\begin{array}{l}0.28 \\
0.01\end{array}$ & $\begin{array}{r}-6.2 \\
2.0\end{array}$ & $\begin{array}{l}3.5 \\
1.5\end{array}$ & $\begin{array}{l}0.071 \\
0.014\end{array}$ & $\begin{array}{l}9.73 \\
0.03\end{array}$ \\
\hline $\begin{array}{l}\text { I TMA } \\
\text { errors }\end{array}$ & 1.216 & $\begin{array}{l}0.34 \\
0.005\end{array}$ & $\begin{array}{l}2.44 \\
0.02\end{array}$ & $\begin{array}{l}0.66 \\
0.005\end{array}$ & $\begin{array}{l}6.66 \\
0.02\end{array}$ & $\begin{array}{l}0.33 \\
0.01\end{array}$ & $\begin{array}{r}-1.6 \\
0.1\end{array}$ & $\begin{array}{r}-2.7 \\
0.2\end{array}$ & $\begin{array}{l}0.118 \\
0.015\end{array}$ & $\begin{array}{l}5.99 \\
0.02\end{array}$ \\
\hline $\begin{array}{l}\text { II TMA } \\
\text { errors }\end{array}$ & 1.265 & $\begin{array}{l}0.34 \\
0.005\end{array}$ & $\begin{array}{l}2.50 \\
0.02\end{array}$ & $\begin{array}{l}0.66 \\
0.005\end{array}$ & $\begin{array}{l}6.65 \\
0.02\end{array}$ & $\begin{array}{l}0.31 \\
0.01\end{array}$ & $\begin{array}{r}-12.2 \\
3.2\end{array}$ & $\begin{array}{l}4.6 \\
1.0\end{array}$ & $\begin{array}{l}0.048 \\
0.011\end{array}$ & $\begin{array}{l}5.99 \\
0.02\end{array}$ \\
\hline
\end{tabular}

a) $\left\langle\tau_{\mathrm{F}}\right\rangle=\int_{0}^{\infty} t F(t) \mathrm{d} t / \int_{0}^{\infty} F(t) \mathrm{d} t$.

Table 2

Order parameters corresponding to the recovered values of $\lambda_{2}$ and $\lambda_{4}$ given in table 1 . Estimated errors in parentheses

\begin{tabular}{llrrrrr}
\hline & \multicolumn{1}{ll}{ POPC } & & \multicolumn{2}{l}{ Cholesterol added } \\
\cline { 2 - 3 } & $\left\langle P_{2}\right\rangle$ & & \multicolumn{1}{l}{$\left\langle P_{2}\right\rangle$} & $\left\langle P_{4}\right\rangle$ \\
\hline I DPH & $0.14(0.03)$ & $0.28(0.01)$ & & $0.50(0.03)$ & $0.42(0.02)$ \\
II DPH & $0.19(0.03)$ & $-0.29(0.04)$ & & $0.53(0.12)$ & $-0.02(0.15)$ \\
I TMA-DPH & $0.51(0.03)$ & $0.42(0.02)$ & & $0.67(0.07)$ & $0.55(0.07)$ \\
II TMA-DPH & $0.56(0.10)$ & $0.01(0.12)$ & & $0.72(0.08)$ & $0.28(0.16)$ \\
\hline
\end{tabular}

into different contributions from the individual functions $G_{k}(t)$ for two distinct orienting potentials, and hence pairs of order parameters. This is illustrated in fig. 3 where the experimental constant $\int_{0}^{\infty} F(t) r(t) \mathrm{d} t$, and the contributions to it from the three correlation functions, are shown as a function of the order parameter $\left\langle P_{4}\right\rangle$ on taking $\left\langle P_{2}\right\rangle=0.5$, and $F(t), D_{\perp}$ and $r(0)$ as given for TMA-DPH in POPC in table 1.

It is interesting to note that the minimum value found for this quantity corresponds closely to the

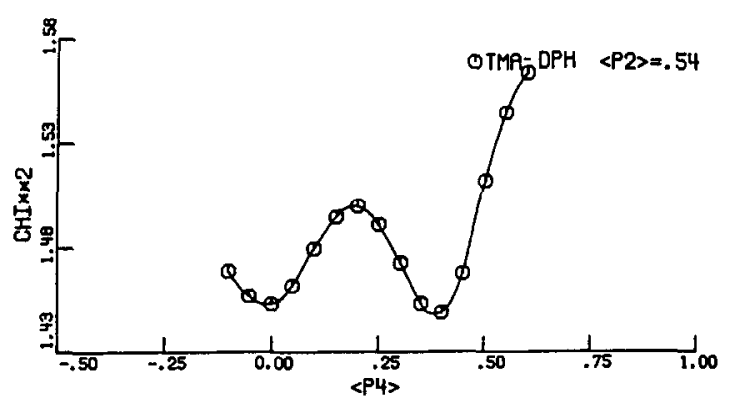

Fig. 2. Minimal reduced $\chi^{2}$ values versus $\left\langle P_{4}\right\rangle$ for TMA-DPH in POPC vesicles. $\left\langle P_{2}\right\rangle=0.54$. 


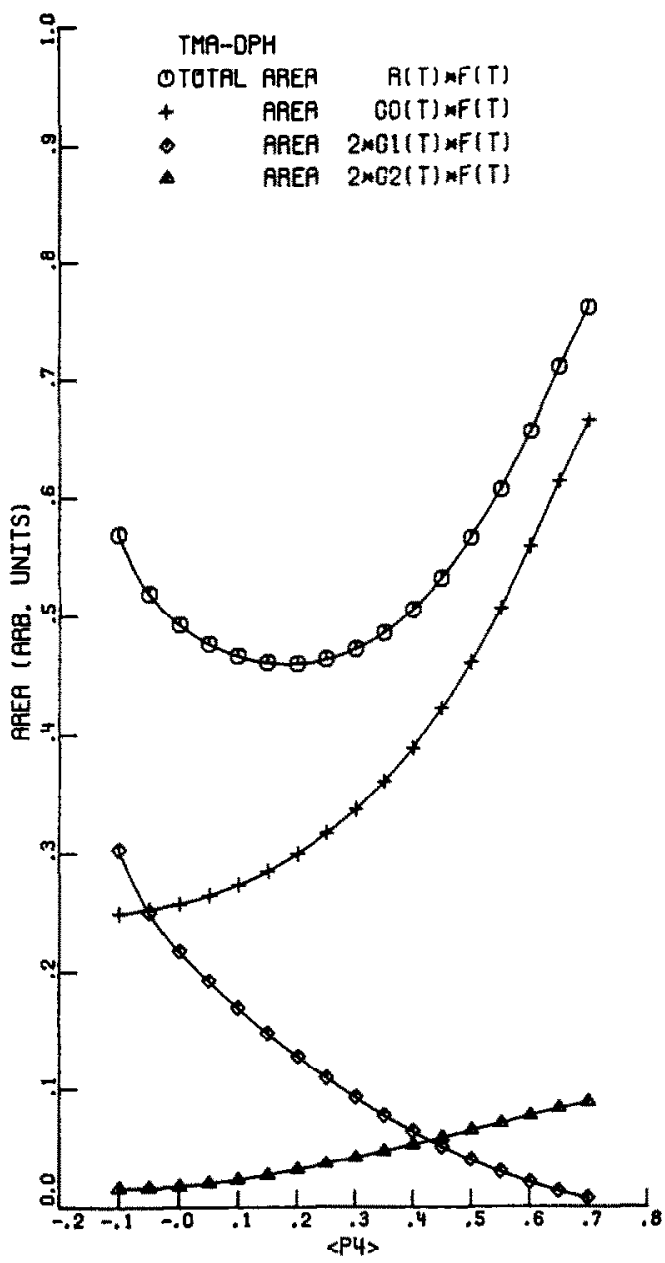

Fig. 3. The total experimental $\int_{0}^{\infty} F(t) r(t) \mathrm{d} t$ and the contributions of the three correlation functions (eq. (2)) as a function of $\left\langle P_{4}\right\rangle$ with $\left\langle P_{2}\right\rangle=0.5$ and $F(t)$ from TMA-DPH in POPC vesicles. $D_{\perp}=0.100 \mathrm{~ns}^{-1}$.

Maier-Saupe potential. Although at this point a unique decomposition is expected, we have shown above that the solution yields a maximum in the $\chi_{\mathrm{r}}^{2}$ surface, fig. 2 .

The question now arises as to which minimum gives the more physically reasonable solution. It is important to note in this connection that the two solutions yield contradictory answers as to the effect of the addition of cholesterol on the dynamics of the probe motion. In minimum I, table 1, an increase of $D_{\perp}$ is found, whereas a decrease is obtained for min-



Fig. 4. Orientational probability distribution functions $f(\beta)$ reconstructed from the two sets of order parameters found for TMA-DPH in POPC vesicles. $\beta$ is the angle between the normal to the bilayer surface and the molecular symmetry axis of the probe.

imum II which corresponds closely to the "wobblein-cone" model [3,7]. Fig. 4 shows the orientational probability distribution functions [12-14] obtained from the orienting potentials at the two minima. The distribution function with the higher $\left\langle P_{4}\right\rangle$ value shows a marked preferential orientation of the long molecular axes along the normal to the local vesicle surface. The solution with the low $\left\langle P_{4}\right\rangle$ values exhibits a collective molecular tilt. An alternative distribution for the latter case is provided by the "wobble-in-cone" model where a uniform distribution over a range of angles is postulated. However, if the probe molecules interdigitate between the lipid chains and reflect their behaviour, then the low $\left\langle P_{4}\right\rangle$ solution cannot be reconciled with the results of other physical techniques $[2,17]$, particularly $X$-ray diffraction $[17,18]$. In marked contrast the other solution does yield a physically reasonable picture of the dynamic structure of the bilayer $[2,17,18]$. Furthermore the distribution is in good agreement with the one obtained from angle-resolved anisotropy measurements on oriented bilayer systems $[12,13]$ in which the individual contributions of the correlation functions $G_{k}(t)$ can be resolved.

\section{Acknowledgement}

The authors wish to thank Ms. M.L. Verheijden for the phosphorus determination and Dr. D. Shaw 
and Dr. G. van Ginkel for their assistance during the experiments. HvL was supported by the Dutch Foundation for Biophysics under the auspices of the Netherlands Organization for Pure Research (ZWO). The use of the SRS was made possible by ZWO in connection with the agreement between SERC and ZWO.

\section{References}

[1] C. Zannoni, A. Arcioni and P. Cavatorta, Chem. Phys. Lipids 32 (1983) 179.

[2] M.A. Hemminga, Chem. Phys. Lipids 32 (1983) 323.

[3] K. Kinosita Jr., S. Kawato and A. Ikegami, Advan. Biophys. 17 (1984) 147.

[4] R.E. Dale, L.A. Chen and L. Brand, J. Biol. Chem. 252 (1977) 7500.

[5] R.B. Cundall and R.E. Dale, Time-resolved fluorescence spectroscopy in biochemistry and biology (Plenum Press, New York, 1983).
[6] M. Ameloot, H. Hendrickx, W. Herreman, H. Pottel, F. van Cauwelaert and W. van der Meer, Biophys. J. 46 (1984) 525.

[7] A. Szabo, J. Chem. Phys. 81 (1984) 150.

[8] W. van der Meer, H. Pottel, W. Herreman, M. Ameloot, H. Hendrickx and H. Schröder, Biophys. J. 46 (1984) 515.

[9] L.V. Natarajan, F.M. Stein, R.E. Blankenship and R. Chang, Chem. Phys. Letters 95 (1983) 525.

[10] M.E. Rose, Elementary theory of angular momentum (Wiley, New York, 1957).

[11] P.L. Nordio and U. Segre, in: The molecular physics of liquid crystals, eds. G.R. Luckhurst and G.W. Gray (Academic Press, New York, 1979) p. 411.

[12] G. van Ginkel, L.J. Korstanje, H. van Langen and Y.K. Levine, Faraday Discussions Chem. Soc. 81 (1986) 49.

[13] F. Mulders, H. van Langen, G. van Ginkel and Y.K. Levine, Biochim. Biophys. Acta 859 (1986) 209.

[14] H. Pottel, W. Herreman, B.W. van der Meer and M. Ameloot, Chem. Phys. 102 (1986) 37.

[15] P.S. Chen Jr., T.Y. Toribara and H. Warner, Anal. Chem. 28 (1956) 1756.

[16] T. Awaya, Nucl. Instr. Methods 165 (1979) 317.

[17] E. Grell, Membrane spectroscopy (Springer, Berlin, 1981). [18] Y.K. Levine, Progr. Biophys. Mol. Biol. 24 (1972) 1. 